\title{
Investigating types and sources of organic aerosol in Rocky Mountain National Park using aerosol mass spectrometry
}

M. I. Schurman et al.

Correspondence to: M. I. Schurman (mishaschurman.ms@gmail.com) 


\section{S1.1 PMF Solution Diagnostics}
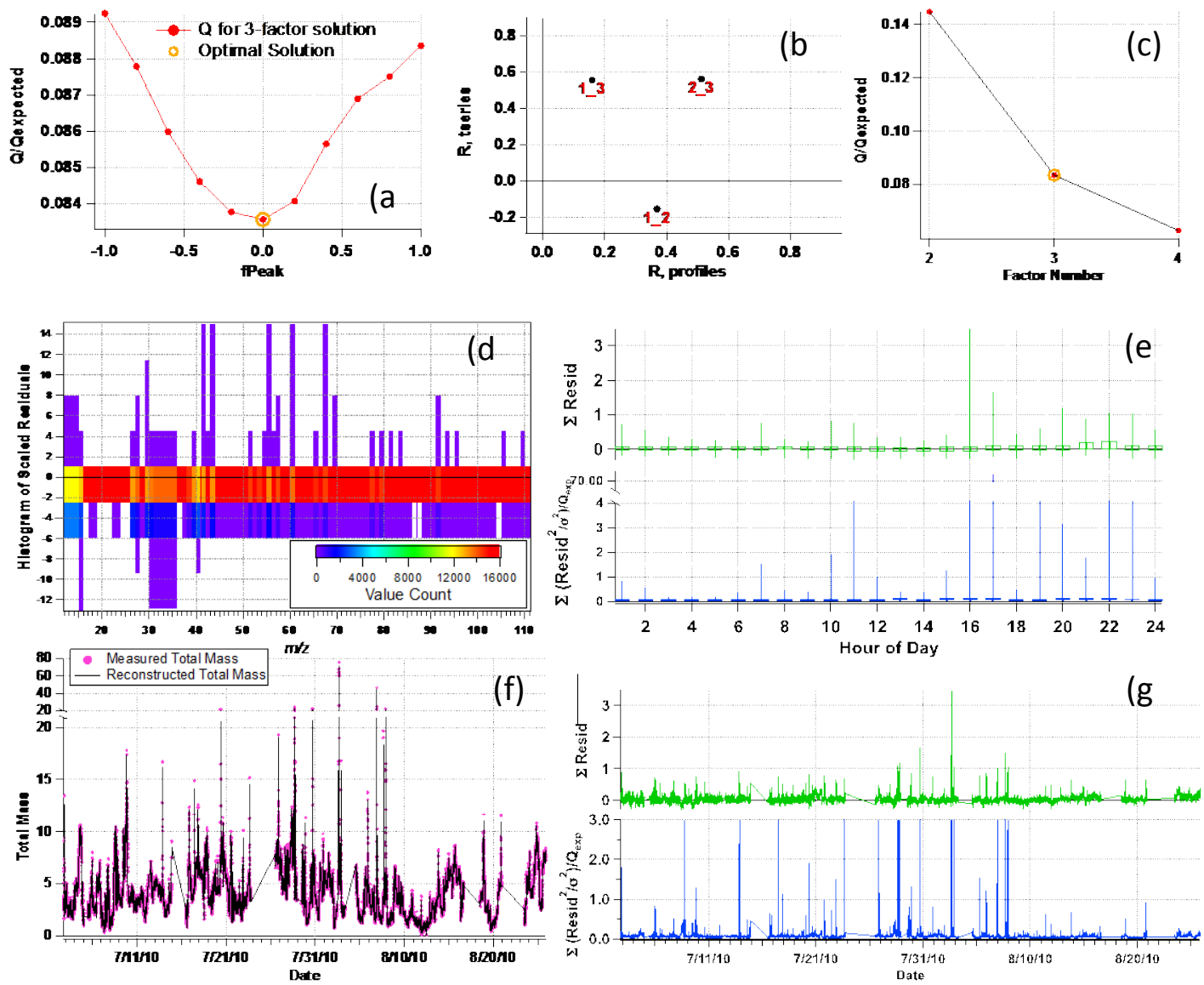

5 Figure S1: Diagnostic plots for the optimal 3-factor PMF solution explored in the main text. (a)

$6 \mathrm{Q} / \mathrm{Q}_{\exp }$ for varying FPEAK values for a 3-factor solution, (b) Pearson's correlation coefficients

7 (R) between factor time series and mass spectral profiles, where Factor $1=$ BBOA, Factor $2=$

8 LV-OOA, and Factor 3 = SV-OOA, (c) Q/Qexp for various numbers of factors, (d) histogram of

9 scaled residuals for each $\mathrm{m} / \mathrm{z}$ (Scaled residual = (measured-reconstructed) $/$ measured), (e) hourly

10 diurnal (top) sum of residuals and (bottom) Q/ $\mathrm{Q}_{\exp }$ (boxes are the mean; whiskers $25^{\text {th }}$ and $75^{\text {th }}$

11 percentiles), (f) total measured and reconstructed mass, and (g) sum of residuals (top) and Q/Q $\mathrm{Qxp}_{\text {p }}$

12 (bottom) over time. 

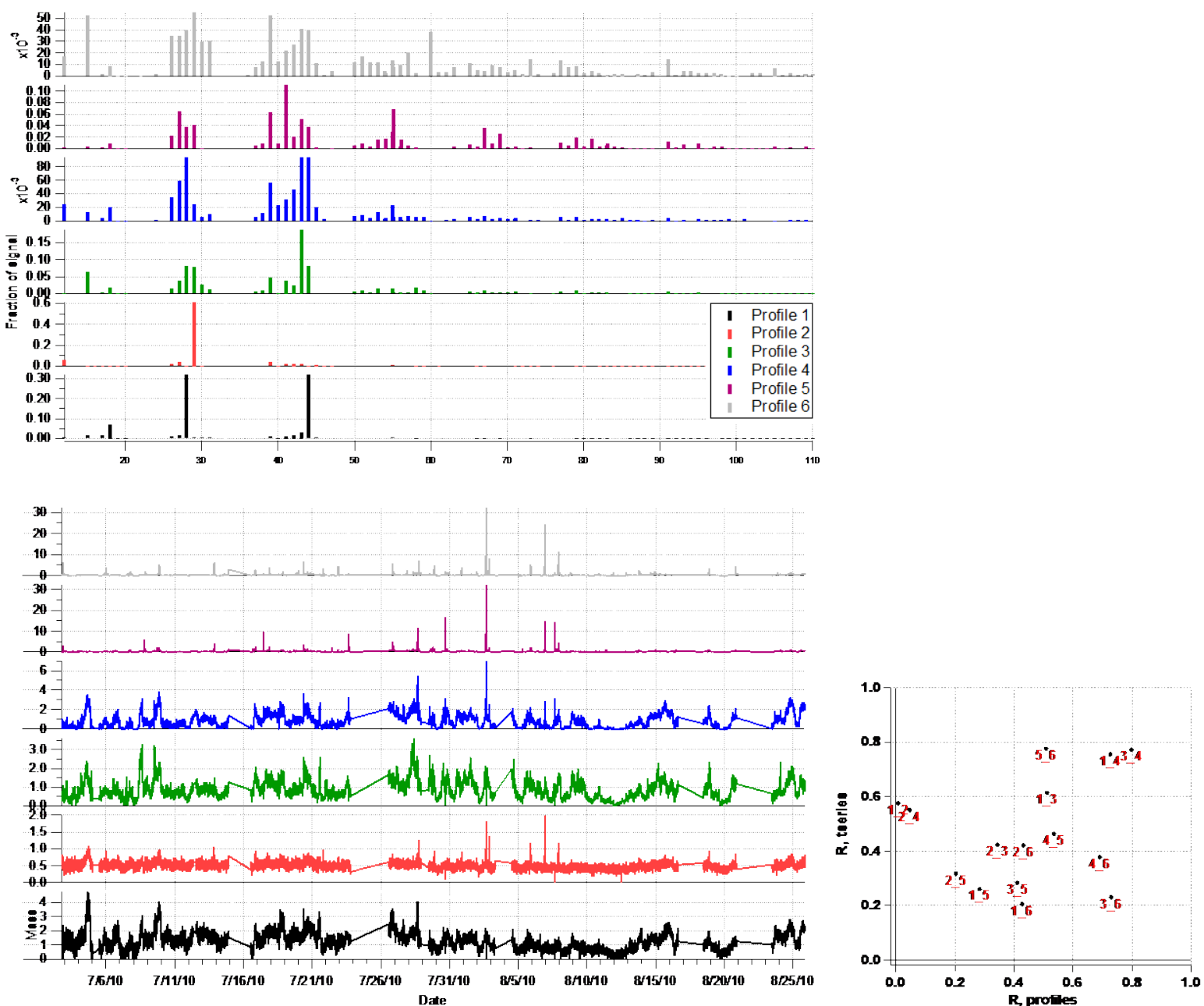

3 Figure S2: (top) Mass spectra, and (bottom) timelines of factors from a 6-factor PMF solution 4 that was subsequently recombined into three factors, and (bottom right) mass spectral and time 5 series correlation coefficients (r) between the six factors.

6 Table S1: Coefficients of determination $\left(\mathrm{r}^{2}\right)$ calculated using the IGOR linear regression 7 algorithm between mass spectral profiles of the three organic factors (*) from the 6-factor 8 recombination.

*SV-OOA

0.31
*SV-OOA

\section{*BBOA}

\subsection{6}

0.47 

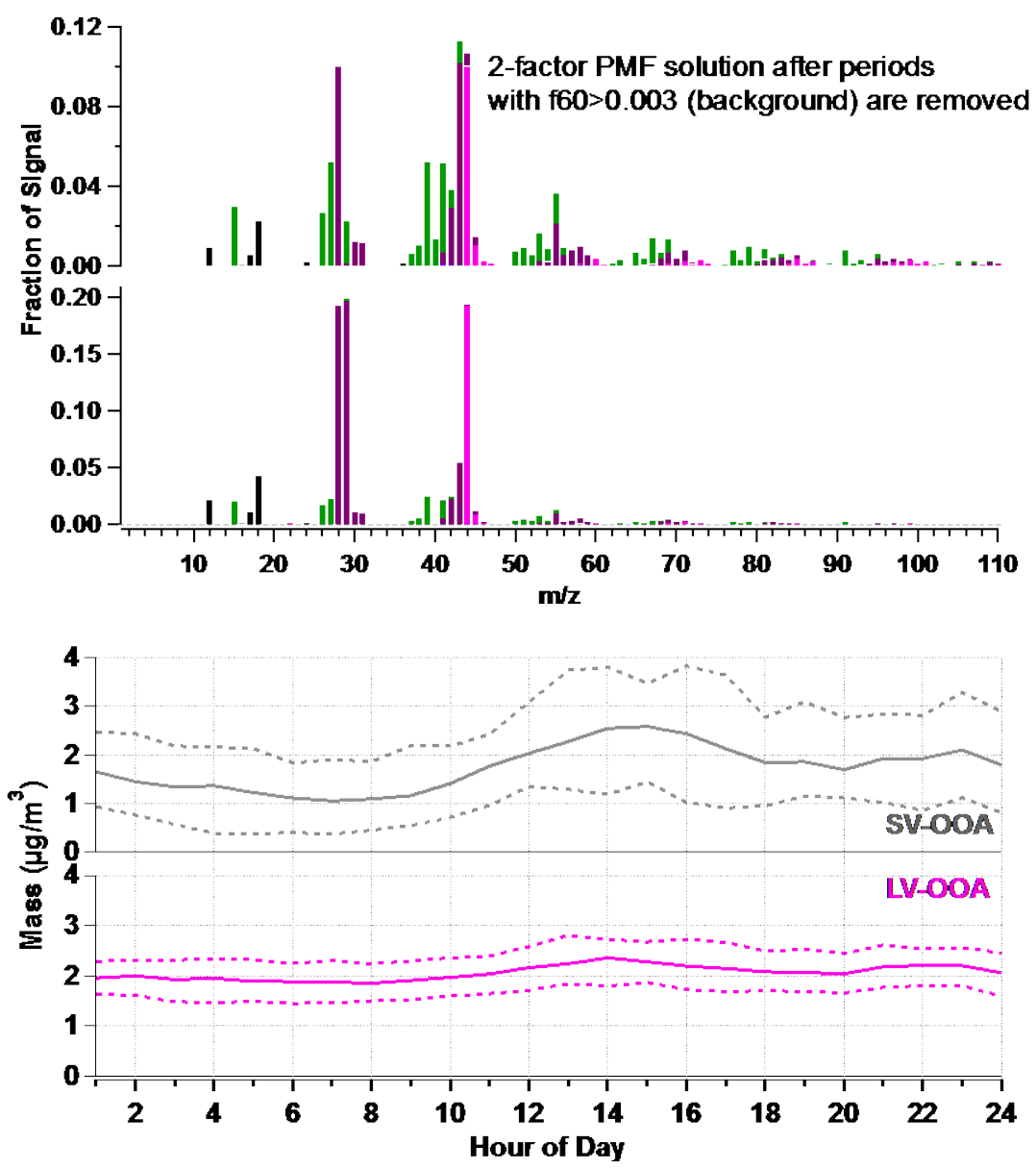

4 Figure S3: (top) Mass spectra and (bottom) diurnal hourly averages of factors from a PMF 5 analysis omitting periods with elevated $f 60$ ( $f 60>0.003$, the ambient background value). 

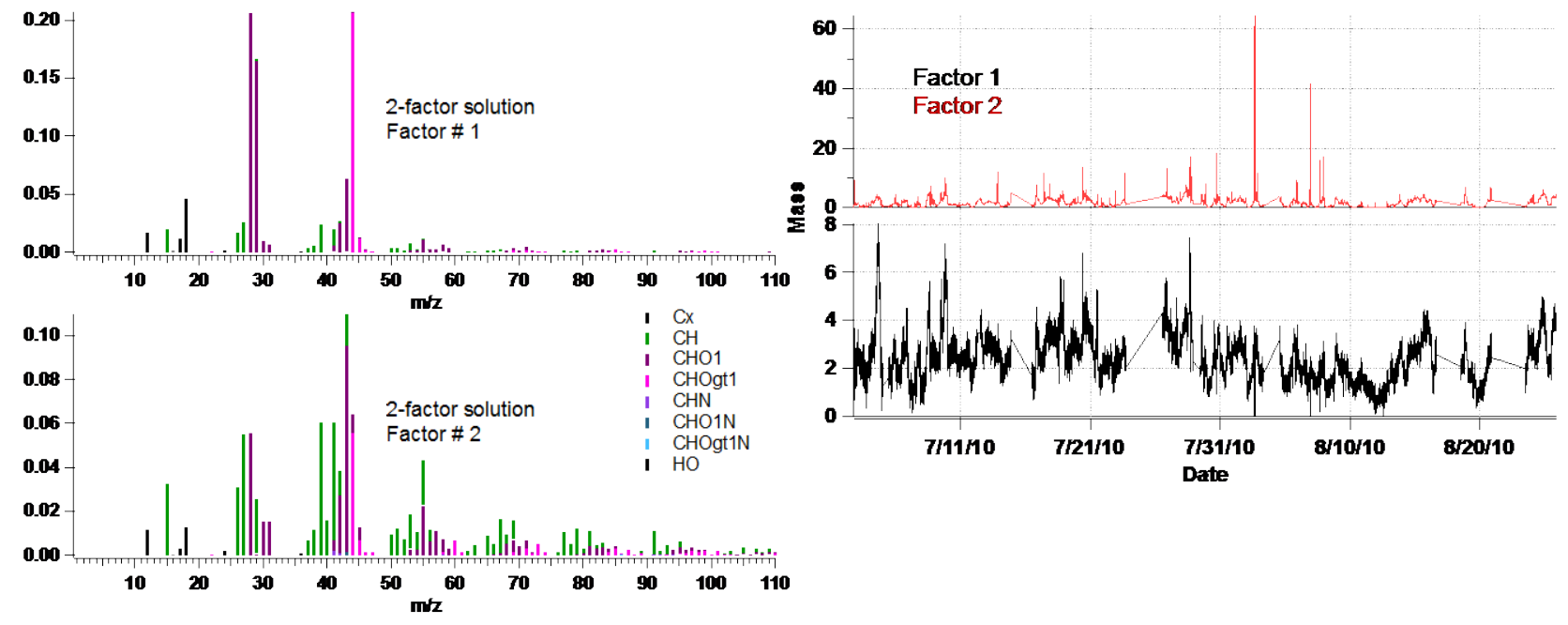

3 Figure S4: A 2-factor PMF solution of the high-resolution organic matrix (no filtering or 4 exclusions of BBOA periods): (left) factor mass spectra colored by fragment family signal 5 contributions, and (right) time series of each factor. 

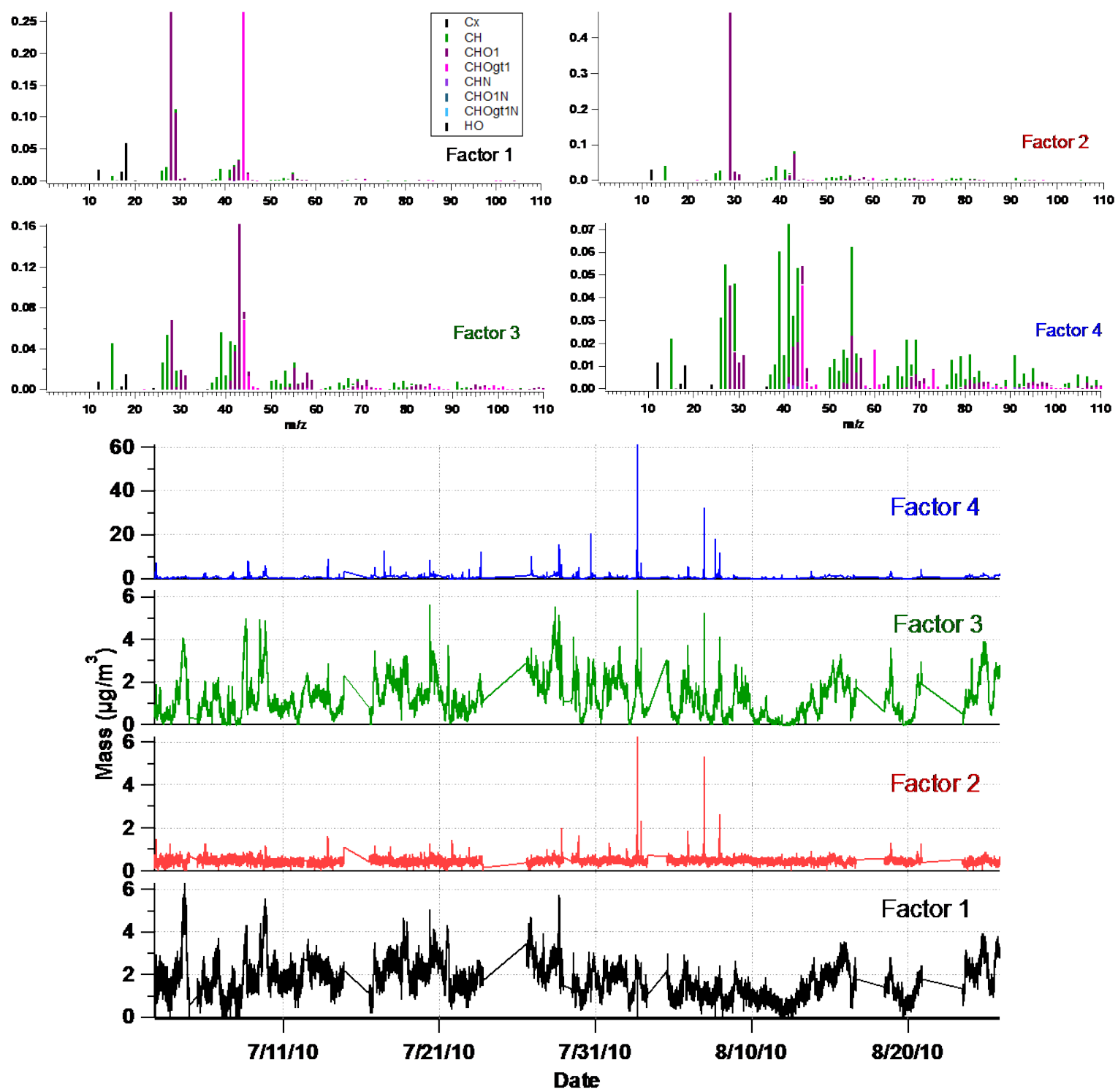

3 Figure S5: A 4-factor PMF solution of the high-resolution organic matrix (no filtering or 4 exclusions of BBOA periods): (top) factor mass spectra colored by fragment family signal 5 contributions, and (bottom) time series of each factor. 


\section{S1.2 Elemental Analysis:}

2 Slopes during periods dominated by each factor are all close to $-0.5\left(\mathrm{~m}_{\mathrm{LV}}=-0.54 ; \mathrm{m}_{\mathrm{SV}}=-0.56\right.$;

$3 \mathrm{~m}_{\mathrm{BB}}=-0.53$ ), consistent with other ambient aerosol studies ( $\mathrm{Ng}$ et al. 2011). The photo-oxidation

4 of $\alpha$-pinene also produces this slope, consistent with possible biogenic contributions, although

5 other reactions can of course yield similar slopes (Chhabra et al. 2011; Lambe et al. 2011); also,

6 because the air masses herein are not isolated, it is also possible that transportcould be

7 influencing the observed changes in van Krevelen space in place of or addition to in-situ reaction.

\section{S1.3 Estimation of PToF Sizing Error:}

9 PToF sizing error should not be confused with PToF mass error calculations in Ulbrich et al.

10 (2012). Sources of error in PToF size determination include, but are not limited to, 'chopper

11 broadening' caused by the spread in particle arrival times caused by the time it takes the chopper

12 slit to pass through beam (particles at end of transmission time enter flight slightly later than ones

13 at beginning); since differently sized particles fly at different velocities, this error is size

14 dependent (Allan et al., 2003). For a $1 \%$ chopper at $150 \mathrm{~Hz}$, the amount of time that the chopper is

15 open, which influences the spread of the sized particles arriving at the vaporizer, can be

16 calculated by:

$17\left(\frac{6.7 \mathrm{~ms}}{\text { revolution }}\right)\left(\frac{0.01 \text { open chopper }}{\text { revolution }}\right)\left(\frac{\text { revolution }}{2 \times 0.5 \% \text { slits }}\right)=0.034 \frac{\mathrm{ms}}{\text { revolution }}$ (Equation 1)

18 For each particle size, the chopper transmission time can be compared to total particle flight time

19 to determine chopper-broadening error. The particle PToF flight time can be calculated via:

$20 \quad$ Velocity $=\mathrm{p}_{3}+\frac{\left(\mathrm{p}_{0}-\mathrm{p}_{3}\right)}{\left(1+\left(\mathrm{D}_{\mathrm{va}} / \mathrm{p}_{1}\right)^{\left.\mathrm{p}_{2}\right)}\right.}$

21 where $\mathrm{p}_{0}=$ velocity of the gas after the aerodynamic lens, $\mathrm{p}_{1}=\mathrm{D}^{*}(\mathrm{~nm})$ coefficient, $\mathrm{p}_{2}=\mathrm{b}$ 22 coefficient, $\mathrm{p}_{3}=$ velocity of the gas in the aerodynamic lens (determined during the size

23 calibration). For example, using size calibration values for the study presented herein, a $300 \mathrm{~nm}$ 24 particle flight time of 0.003136 seconds yields an error of $0.000034 \mathrm{~s} / 0.003136 \mathrm{~s}=0.011$ or $251.1 \%$.

26 Chopper broadening error is exacerbated by error in PSL or DMA-selected ammonium nitrate used for sizing (error about the size calibration curve); since these errors have different units, each is calculated as a percentage of diameter at each given size and compounded via: 
1 Total Error (\% of diameter) $=\sqrt{\sum_{\mathrm{i}} \mathrm{e}_{\mathrm{i}}^{2}}$

(Equation 3)

2 where e is the \% error for each relevant process. Polystyrene latex spheres (PSL, Duke Scientific 3 Corp.) are used for size calibration points at 70, 100, 200, 300, 400, 500, and $700 \mathrm{~nm}$, and have 4 precisions of 1.5-9 nm, depending on size. For example, 300nm PSL particles have a diameter 5 standard deviation of $5 \mathrm{~nm}$ or 0.0167 (1.7\%) of particle size. Compounding chopper broadening 6 and calibration errors yields a total 2.7\% error in size determination for $300 \mathrm{~nm}$ particles during 7 this study; these calculations were iterated at the diameters listed above for each field experiment. 8 Other possible PToF errors and caveats to a complete PToF error determination are outlined in 9 Ulbrich et al. (2012). The PToF size resolution is 5-10 Daero/ $\Delta \mathrm{D}_{\text {aero }}$ (FWHM) over the size range 10 of aerodynamic lens transmission (Aerodyne Research Inc. 2004). 